ADP-01-58/T490

\title{
Cooling for instantons and the Wrath of Nahm
}

\author{
S. Bilson-Thompson, F. D. R. Bonnet, D. B. Leinweber, and A. G. Williams ${ }^{\text {a }}$ \\ ${ }^{a}$ Centre for the Subatomic Structure of Matter and Department of Physics and Mathematical Physics, \\ University of Adelaide, Adelaide 5005, Australia
}

The dynamics of instantons and anti-instantons in lattice QCD can be studied by analysing the action and topological charge of configurations as they approach a self-dual or anti-self-dual state, i.e. a state in which $S / S_{0}=|Q|$. We use cooling to reveal the semi-classical structure of the configurations we study. Improved actions which eliminate discretization errors up to and including $\mathcal{O}\left(a^{4}\right)$ are used to stabilise instantons as we cool for several thousand sweeps. An analogously improved lattice version of the continuum field-strength tensor is used to construct a topological charge free from $\mathcal{O}\left(a^{4}\right)$ discretization errors. Values of the action and topological charge obtained with these improved operators approach mutually-consistent integer values to within a few parts in $10^{4}$ after several hundred cooling sweeps. Analysis of configurations with $|Q| \approx 1$ and $|Q| \approx 2$ supports the hypothesis that a self-dual $|Q|=1$ configuration cannot exist on the 4-torus.

\section{INSTANTONS}

In 1975 Belavin et al. [1] determined that the vacuum of QCD contained non-trivial local minima of the Euclidean action,

$S_{E}=\frac{1}{2 g^{2}} \int d^{4} x \operatorname{Tr} F_{\mu \nu} F_{\mu \nu}$.

Since this action is required to be finite in the case of semi-classical solutions (i.e. solutions obtained by minimizing the action) the definition of the field-strength tensor

$F_{\mu \nu}=\partial_{\mu} A_{\nu}-\partial_{\nu} A_{\mu}+\left[A_{\mu}, A_{\nu}\right]$

implies that

$A_{\mu} \longrightarrow U^{-1} \partial_{\mu} U$

as $|x|$ goes to infinity. The gauge transformation $U$ is interpreted as a mapping from $S^{3} \longrightarrow S U(2)$, that is, a mapping from the sphere at spacial infinity to the gauge group $S U(2)$ of the transformation $U(S U(2)$ results can be readily extended to $S U(3))$. The mapping $U$ can then be shown to have a winding number given by the equation

$n=\frac{1}{16 \pi^{2}} \int d^{4} x \operatorname{Tr} F_{\mu \nu} \widetilde{F}_{\mu \nu}$

and hence we can associate a winding number with each vacuum state. Instantons are interpreted as tunnelling amplitudes between vacuum states with different winding numbers, and their topological charge is given by the difference between the winding numbers of the states that they connect. Further important properties of (anti-)instantons are spherical symmetry in the four dimensions of Euclidean spacetime, topological charge of \pm 1 , and an associated action of $S_{0}=8 \pi^{2} / g^{2}$ which is independent of the radius of the instanton.

\section{CONNECTING THE LATTICE TO THE CONTINUUM}

The lattice is a 4-dimensional approximation to Euclidean spacetime. This means that physics on the lattice should ideally approach physics in the continuum if we can take the limit of infinite lattice volume and infinitessimal lattice spacing $(V \longrightarrow \infty$ and $a \longrightarrow 0)$. Unfortunately reducing the lattice spacing indefinitely is not practical, nor is increasing the total lattice volume, since these approaches to the continuum limit rapidly increase the time taken to perform computations. We have chosen to remove the non-physical 'edge' of the lattice (simulating an infinite volume) by defining periodic untwisted boundary conditions on our lattices. We are therefore performing numerical QCD on a 4-toroidal mesh of points. 
The discretization errors introduced by the finite lattice spacing are reduced algebraically by Symanzic improvement [2] and non-classical errors arising from the self-couplings of the gluon fields are dealt with by the use of mean-field improvement [3].

Since the lattice we use is topologically equivalent to a 4-torus, we may consider the consequences of the Nahm transform [4 for the stability of the configurations we study. The Nahm transform is a duality mapping which interchanges between an $S U(N)$ configuration with topological charge $Q$ on the torus and an $S U(Q)$ configuration with topological charge $N$ on the dual torus. Since there are no instanton solutions in $U(1)$, the Nahm transform implies [5] [6] that there can be no self-dual $|Q|=1$ configurations on the torus.

\section{THE LATTICE ACTION AND TOPO- LOGICAL CHARGE}

\subsection{The Wilson Action}

The Yang-Mills action on the lattice [7] is

$S_{\mathrm{Wil}}=\beta \sum_{x} \sum_{\mu<\nu}\left[1-\frac{1}{N}\left(\operatorname{Re} \operatorname{Tr} W_{\mu \nu}^{(1 \times 1)}(x)\right)\right]$

where $W_{\mu \nu}^{(m \times n)}$ is the $m \times n$ Wilson loop in the $\mu-\nu$ plane. When we expand the plaquette $W_{\mu \nu}^{(1 \times 1)}$ around the point $x$ and set $\beta=6 / g^{2}$ it can be easily shown that (5) approaches the continuum Yang-Mills action, plus $\mathcal{O}\left(a^{2}\right)$ corrections

$S_{\mathrm{Wil}} \longrightarrow \frac{1}{2} \int d^{4} x \operatorname{Tr} F_{\mu \nu} F_{\mu \nu}+\mathcal{O}\left(a^{2}\right)$

in the continuum limit $a \longrightarrow 0$.

\subsection{Improving the Action}

We can eliminate $\mathcal{O}\left(a^{2}\right)$ errors by constructing the Wilson action from a linear combination of the plaquette and the average of the $1 \times 2$ and $2 \times 1$ rectangular Wilson loops. We may of course choose to employ other loops to eliminate higherorder error terms. DeForcrand et al. [8] have previously constructed an action free from $\mathcal{O}\left(a^{2}\right)$ and $\mathcal{O}\left(a^{4}\right)$ errors by using the five planar Wilson loops

$$
\text { - } L^{(1,1)}=W_{\mu \nu}^{(1 \times 1)}
$$

- $L^{(2,2)}=W_{\mu \nu}^{(2 \times 2)}$

- $L^{(1,2)}=\frac{1}{2}\left(W_{\mu \nu}^{(1 \times 2)}+W_{\mu \nu}^{(2 \times 1)}\right)$

- $L^{(1,3)}=\frac{1}{2}\left(W_{\mu \nu}^{(1 \times 3)}+W_{\mu \nu}^{(3 \times 1)}\right)$.

- $L^{(3,3)}=W_{\mu \nu}^{(3 \times 3)}$

A general improved action can then be written in the form

$$
\begin{aligned}
S_{\operatorname{Imp}}= & c_{1} S\left(L^{(1,1)}\right)+c_{2} S\left(L^{(2,2)}\right)+c_{3} S\left(L^{(1,2)}\right) \\
& c_{4} S\left(L^{(1,3)}\right)+c_{5} S\left(L^{(3,3)}\right) .
\end{aligned}
$$

where the $c_{1}, \ldots, c_{5}$ are improvement factors which take the values

$$
\begin{aligned}
& c_{1}=\left(19-55 c_{5}\right) / 9 \\
& c_{2}=\left(1-64 c_{5}\right) / 9 \\
& c_{3}=\left(640 c_{5}-64\right) / 45 \\
& c_{4}=1 / 5-2 c_{5}
\end{aligned}
$$

and $c_{5}$ is a free parameter which we can use to "tune" the action. By setting $c_{5}=0$ we create a 4-loop improved action, denoted 4LIS for short, and by setting $c_{5}=1 / 10$ we eliminate the contribution of $S\left(L^{(1,2)}\right)$ and $S\left(L^{(1,3)}\right)$, thereby creating a 3-loop improved action, denoted 3LIS for short. For the purposes of investigating a 5LIS we choose to follow DeForcrand et al. and set $c_{5}=1 / 20$, midway between the 3LIS and 4LIS values. It is important to note that the 3LIS and 4LIS are just special cases of the general 5LIS, and all choices of $c_{5}$ are free from discretization errors up to and including $\mathcal{O}\left(a^{4}\right)$, however their $\mathcal{O}\left(a^{6}\right)$ errors will be different, leading to slightly different results.

\subsection{Topological Charge}

On the lattice the topological charge is calculated as the sum of the local topological charge density over all lattice sites,

$Q=\sum_{x} q(x)=\frac{1}{32 \pi^{2}} \sum_{x} \epsilon_{\mu \nu \rho \sigma} \operatorname{Tr} F_{\mu \nu} F_{\rho \sigma}$.

If we have a dilute instanton gas with $n_{I}$ instantons and $n_{A}$ anti-instantons the total topological charge of the configuration will be

$Q=n_{I}-n_{A}$. 
We hence desire the topological charge calculated on the lattice to approach integer values as the configuration being studied is cooled towards a dilute instanton gas condition. Unfortunately discretization errors tend to lead to non-integer values of $Q$. Fortunately, however, we can improve the topological charge.

\subsection{The Improved Field-Strength Tensor}

To improve the topological charge we have chosen to improve the field-strength tensor $F_{\mu \nu}$ directly, by analogy with the improvement of the action, and substitute this into Eq. (8), the definition of the topological charge.

Consider the full expansion of the plaquette

$$
\begin{aligned}
W_{\mu \nu}^{(1 \times 1)}= & e^{i g \oint A d x} \\
= & 1+i g \oint A d x-\frac{g^{2}}{2}(\oint A d x)^{2} \\
& +\mathcal{O}\left(g^{3}\right) \\
= & 1+i g\left[a^{2} F_{\mu \nu}+\mathcal{O}\left(a^{4}\right)\right]-\frac{g^{2} a^{4}}{2} F_{\mu \nu}^{2} \\
& +\mathcal{O}\left(a^{6}, g^{3}\right) .
\end{aligned}
$$

We wish to extract the second term (within the square parentheses) on the last line. This may be achieved by making the following construction;

$$
\begin{aligned}
W_{\mu \nu}^{(1 \times 1)}= & 1+i g \oint A d x-\frac{g^{2}}{2}(\oint A d x)^{2} \\
& +\mathcal{O}\left(g^{3}\right), \\
W_{\mu \nu}^{(1 \times 1) \dagger}= & 1-i g \oint A d x-\frac{g^{2}}{2}(\oint A d x)^{2} \\
& +\mathcal{O}\left(g^{3}\right) .
\end{aligned}
$$

Writing

$$
\mathcal{A}=W_{\mu \nu}^{(1 \times 1)}-W_{\mu \nu}^{(1 \times 1) \dagger}
$$

we may obtain a term proportional to the field strength

$$
\begin{aligned}
\Rightarrow \frac{-i}{2}\left(\mathcal{A}-\frac{1}{3} \operatorname{Tr} \mathcal{A}\right) & =g \oint A d x+\mathcal{O}\left(g^{3}\right) \\
& =g a^{2} F_{\mu \nu}+\mathcal{O}\left(g a^{4}\right) .
\end{aligned}
$$

Notice that in order to enforce the tracelessness of the Gell-Mann matrices we have subtracted onethird of the trace of $\mathcal{A}$.
To construct an improved field strength tensor we utilise the same five planar Wilson loops that are used in the construction of the improved action, but in this case we calculate $W_{\mu \nu}^{(m \times n)}$ from the clover average of four $m \times n$ Wilson loops in the $\mu-\nu$ plane. The improved field strength tensor can then be written

$$
\begin{aligned}
F_{\mu \nu(\operatorname{Imp})}= & k_{1} F_{\mu \nu}\left(L^{(1,1)}\right)+k_{2} F_{\mu \nu}\left(L^{(2,2)}\right) \\
& +k_{3} F_{\mu \nu}\left(L^{(1,2)}\right)+k_{4} F_{\mu \nu}\left(L^{(1,3)}\right) \\
& +k_{5} F_{\mu \nu}\left(L^{(3,3)}\right)
\end{aligned}
$$

where the $k_{1}, \ldots, k_{5}$ are improvement factors which take the values

$$
\begin{aligned}
k_{1} & =19 / 9-55 k_{5} \\
k_{2} & =1 / 36-16 k_{5} \\
k_{3} & =64 k_{5}-32 / 45 \\
k_{4} & =1 / 15-6 k_{5}
\end{aligned}
$$

and $k_{5}$ is a free parameter, which enables us to create 3-loop, 4-loop, and 5-loop improved definitions of the field-strength, all of which are $\mathcal{O}\left(a^{4}\right)$ improved.

In addition to constructing $Q$, the field strength may be used in (5) to create a "reconstructed action". Since this action is improved in a different manner to the cooling action, comparison of both actions may serve as a mechanism with which to test the improvement procedures.

In this brief report we will simply "test the water" with the values $c_{5}=k_{5}=1 / 20$ (5-loop), $c_{5}=$ $k_{5}=0$ (4-loop), and $c_{5}=1 / 10, k_{5}=1 / 90$ (3loop), and show that we obtain excellent results even though many other 5-loop operators, corresponding to other choices of $c_{5}$ and $k_{5}$, are possible.

\section{RESULTS AND COMPARISON}

\subsection{Constructing Configurations}

The configurations used in our investigations are generated using the Cabibbo-Marinari pseudo-heatbath algorithm [9]. We generate $S U(3)$ configurations by looping twice over three diagonal $S U(2)$ subgroups. From a cold start (all link values set to zero) we thermalize for 5000 sweeps using an $\mathcal{O}\left(a^{2}\right)$-improved action and a 


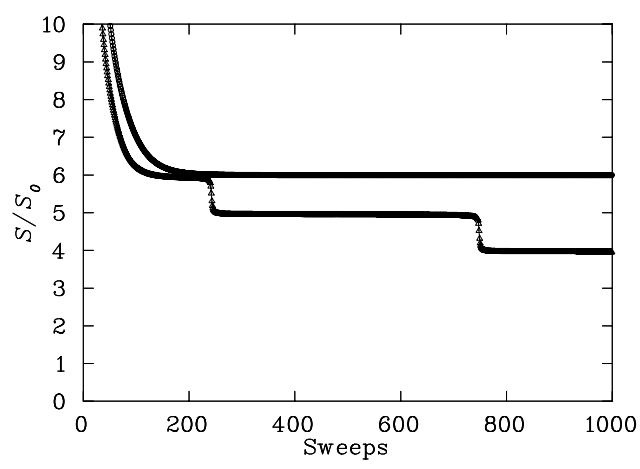

Figure 1. Action obtained with 1-loop $(\triangle)$ and 2-loop (o) cooling.

fixed mean-link value, and then select configurations every 500 sweeps to create an ensemble of configurations with non-trivial topology, which are statistically distinct from each other.

To reveal the semi-classical structure of the configurations we use a cooling algorithm which likewise uses diagonally embedded $S U(2)$ subgroups, with appropriate link partitioning 10.

The results we present are produced from configurations on a $12^{3} \times 24$ lattice with $\beta=4.60$, providing a lattice spacing of $a=0.125 \mathrm{fm}$.

\subsection{Comparison of Cooling Schemes}

In practice, because of the large discretization errors present, a cooling scheme based upon the plaquette action will destroy topological structure over the whole lattice if it proceeds for long enough. However, a cooling scheme using an improved action will stabilise at a state with non-trivial structure. We would anticipate cooling schemes with small discretization errors will reproduce expected continuum behaviour, especially integer values of $Q$ and $S / S_{0}$, and remain stable for hundreds of cooling sweeps. When comparing several cooling schemes, the one which fulfills these criteria best will be the preferred scheme for studying gauge field dynamics.

In Figure 11 we can see how the use of a 2loop, $\mathcal{O}\left(a^{2}\right)$-improved action in the cooling algo-

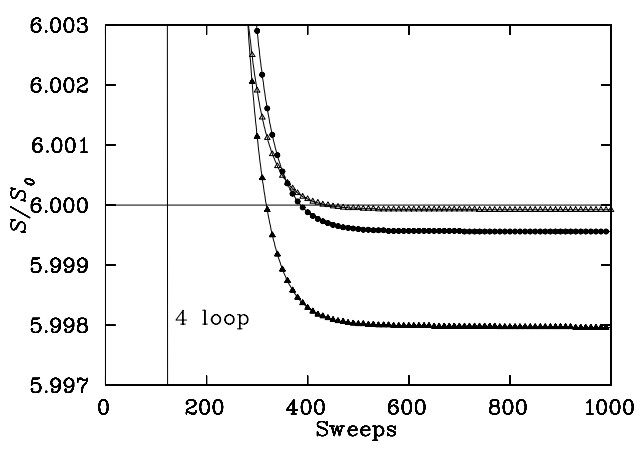

Figure 2. Action obtained with 2-loop ( $\mathbf{\Delta})$, 3LIS $(\triangle)$, LLIS (marked), and 5LIS $(\bullet)$ cooling.

rithm stabilises a configuration at a state with $S / S_{0} \approx 6$, indicating the presence of six instantons and/or anti-instantons, while plaquettebased cooling gradually destroys the structure of the configuration.

In Figure 2 we compare the values of the action obtained with 2-loop, 3LIS, 4LIS, and 5LIS-based cooling on the same configuration used in Figure 1. We can see that the 4LIS curve drops well below the desired integer value of six, indicating the presence of large negative discretization errors. This comparison and similar work with other configurations have lead us to conclude that 3LIS-based cooling gives the most integer-like results in general, and furthermore it is faster than 4LIS or 5LIS-based cooling, since it requires fewer computational steps.

\subsection{Comparison of Topological Charge Improvement}

In Figure 3 we compare the various improved $Q$ values obtained as we cool a configuration. The 3-loop improved operator gives the most continuum-like results, coming to within around 1 part in 6000 of an integer, and has the smallest computational cost of all the $\mathcal{O}\left(a^{4}\right)$-improved operators. Similar comparisons with other configurations have lead us to select the 3-loop operator as our preferred choice for studying the topological charge of cooled configurations. 


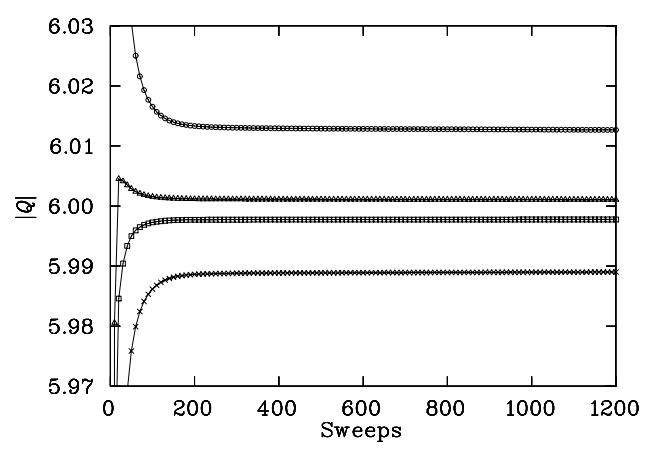

Figure 3. $|Q|$ of $2 \mathrm{~L}(\times), 3 \mathrm{~L}(\triangle), 4 \mathrm{~L}(\square)$, and $5 \mathrm{~L}$ (०), with 3LIS cooling

\subsection{Investigation of the Nahm Transform}

Having established our preferred operators for investigating instanton dynamics, we are now in a position to compare the stability of $|Q|=1$ and $|Q| \neq 1$ configurations, to determine if we can find any evidence that self-dual $|Q|=1$ configurations are not permitted on the 4-torus, as implied by the Nahm transform. Figure 1 shows an overlay of the reconstructed action and topological charge of CFG 1, a $|Q| \approx 1$ configuration, and the corresponding values for CFG 2 , a $|Q| \approx 2$ configuration (shifted down by an increment of one unit so that they overlap with those of CFG 1 on the scale of this diagram). These configurations are separated by 8000 thermalization sweeps. We can clearly see that the $|Q| \approx 2$ configuration stabilises quickly at a self-dual near-integer value, while the other configuration does not achieve self-duality and eventually destabilises. Investigations of other configurations have shown equivalent behaviour.

\section{CONCLUSIONS}

Highly improved operators enable us to cool configurations to stable non-trivial self-duality. Counting the number of instantons and antiinstantons present by evaluating $S / S_{0}$ and $Q$ allows us to detect evidence of continuum-like behaviour. The long-term stability of $|Q| \approx 2$ configurations indicates that our cooling algorithms

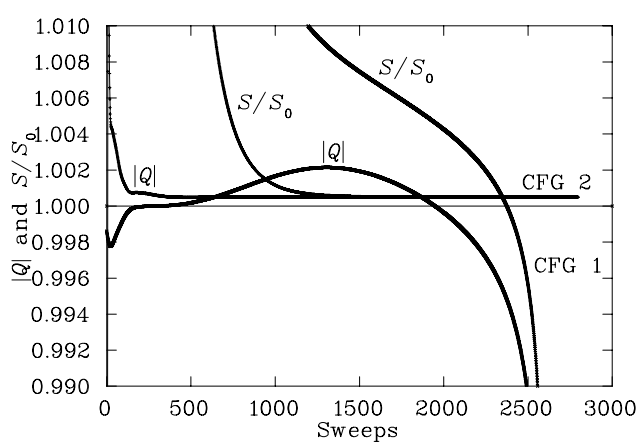

Figure 4. 3-loop improved $|Q|$ and reconstructed $S / S_{0}$ of CFG 1 and CFG 2

are highly reliable, and hence the destabilization of $|Q| \approx 1$ configurations is due to the consequences of the Nahm transform, indicating that on the lattice, just as in the continuum, a configuration may not simultaneously fulfill the two criteria $S / S_{0}=1$ and $|Q|=1$ on the 4 -torus.

\section{REFERENCES}

1. A. A. Belavin, A. M. Polyakov, A. S. Schwartz and Yu. S. Tyupkin, Phys. Lett B59 85 (1975).

2. K. Symanzic Nucl. Phys B226 187 (1983).

3. G. P. LePage, Phys.Rev. D48 22502264 (1993).

4. A. Gonz'alez-Arroyo and C. Pena, Nucl. Phys. Proc. Suppl. 83 533-535 (2000).

5. C. Taubes, J. Diff. Geom. 19, 517-560 (1984).

6. H. Schenk, Comm. Math. Phys. 116, 177-183 (1988).

7. K. G. Wilson, Phys. Rev. D10 2445 (1974).

8. P. DeForcrand, M. G. Perez, I-O. Stamatescu Nucl. Phys B499 409 (1997).

9. N. Cabibbo and E. Marinari, Phys. Lett B119 387 (1982).

10. F. D. R.Bonnet, D. B. Leinweber, and A. G. Williams, J. Comput. Phys. 170 1-17 (2001). 